\title{
PET/MRI: THE NEXT GENERATION OF MULTI-MODALITY IMAGING?
}

\author{
Bernd Pichler, \\ Laboratory for Preclinical Imaging and Imaging Technology of the Werner Siemens-Foundation, \\ Department of Radiology, University of Tübingen, Tübingen, Germany
}

Hans F Wehrl, Laboratory for Preclinical Imaging and Imaging Technology of the Werner Siemens-Foundation, Department of Radiology, University of Tübingen, Tübingen, Germany

Armin Kolb, and Laboratory for Preclinical Imaging and Imaging Technology of the Werner Siemens-Foundation, Department of Radiology, University of Tübingen, Tübingen, Germany

Martin S Judenhofer

Laboratory for Preclinical Imaging and Imaging Technology of the Werner Siemens-Foundation, Department of Radiology, University of Tübingen, Tübingen, Germany

\section{Abstract}

Multi-modal imaging is now well-established in routine clinical practice. Especially in the field of Nuclear Medicine, new PET installations are comprised almost exclusively of combined PET/CT scanners rather than PET-only systems. However, PET/CT has certain notable shortcomings, including the inability to perform simultaneous data acquisition and the significant radiation dose to the patient contributed by CT. MRI offers, compared to CT, better contrast among soft tissues as well as functional-imaging capabilities. Therefore, the combination of PET with MRI provides many advantages which go far beyond simply combining functional PET information with structural MRI information. Many technical challenges, including possible interference between these modalities, have to be solved when combining PET and MRI and various approaches have been adapted to resolving these issues. Here we present an overview of current working prototypes of combined PET/ MRI scanners from different groups. In addition, besides PET/MR images of mice, the first such images of a rat PET/MR, acquired with the first commercial clinical PET/MRI scanner, are presented. The combination of PET and MR is a promising tool in pre-clinical research and will certainly progress to clinical application.

\section{INTRODUCTION}

The ability to achieve millimeter or even sub-millimeter spatial resolution with the latest $\mathrm{x}$-ray computer tomographs (CT) and magnetic resonance imaging (MRI) scanners has propelled

(C) 2008 Elsevier Inc. All rights reserved.

Corresponding author: Prof. Dr. Bernd Pichler, PhD Laboratory for Preclinical Imaging and Imaging Technology of the Werner SiemensFoundation Department of Radiology University of Tübingen Röntgenweg 1372076 Tübingen, Germany Telephone Number: +49-7071-29-83427 Fax Number: +49-7071-29-4451 E-mail Address: Bernd.Pichler@med.uni-tuebingen.de.

Publisher's Disclaimer: This is a PDF file of an unedited manuscript that has been accepted for publication. As a service to our customers we are providing this early version of the manuscript. The manuscript will undergo copyediting, typesetting, and review of the resulting proof before it is published in its final citable form. Please note that during the production process errors may be discovered which could affect the content, and all legal disclaimers that apply to the journal pertain. 
non-invasive imaging into a new generation of advanced applications. Nuclear Medicine imaging, particularly positron emission tomography (PET), has dramatically progressed as well. The uniquely high sensitivity of PET - in the picomolar range - allows detection of even minute amounts of radiolabeled markers in vivo, making PET the modality of choice for molecular imaging. The commercial availability of ${ }^{18} \mathrm{~F}$-labeled tracers such as ${ }^{18} \mathrm{~F}$ fluorodeoxyglucose $\left(\left[{ }^{18} \mathrm{~F}\right] \mathrm{FDG}\right)$ helped stimulate the widespread introduction of PET in private practices and smaller hospitals and medical centers. An important new perspective in the field of nuclear imaging was created by the introduction of combined PET/CT in 2001 $(1,2)$. The ability to accurately and transparently combine functional PET information with high-resolution, three-dimensional morphological CT information with a single device established a clear new direction for diagnostic imaging: accruing as much information as possible with a single examination. Compared to two stand-alone machines, the combination of a PET and CT scanner into a single gantry provides not only greater convenience, flexibility, and speed for multi-modality imaging but also simplifies the logistics of patient management significantly. Furthermore, it ensures far greater accuracy in registration of PET and CT data than could be achieved with software-based fusion of separately acquired imaging studies. The fact that the sales of PET scanners increased world-wide by nearly ten-fold over the last eight years demonstrates the importance of functional nuclear imaging in clinical diagnosis. Today, in fact, all newly purchased and installed PET scanners are combined PET/CT devices and a number of studies have clearly shown advantages of such studies over CT or PET examinations alone or PET/CT carried out using two stand-alone machines (3-6). Early in the clinical development of PET/CT, only low-dose CT scans were performed and were used to anatomically localize PET findings and to derive more accurate attenuation corrections maps and thus improve PET-based activity quantitation. More recently, however, it has been demonstrated that a full, diagnostic-quality CT scan significantly improves the overall clinical value of PET/CT studies (7) - at the cost, however, of a higher CT dose to the patient.

\section{THE LIMITATIONS OF PET/CT AND THE POTENTIAL OF PET/MRI}

Although PET/CT is rapidly becoming a critical component of clinical diagnoses, it has certain limitations, largely related to the fact that the $\mathrm{CT}$ and PET scans are acquired sequentially rather than simultaneously. Especially problematic are artefacts caused by intra- and inter-scan patient and organ motion and differences between the breathing protocols used in PET and CT. These artefacts affect the accuracy of the registration and attenuation correction, seriously compromising the accuracy of activity quantitation (8-11). In addition, there are many clinical indications where MRI is preferred over CT (12-14). The excellent soft-tissue contrast and the fact that it does not use ionizing radiation are additional notable advantages of MRI both clinically and pre-clinically (15). Thus, at about the same time developmental work on PET/ CT scanners began, work was also initiated on combined PET/MRI devices $(2,16,33)$. While full integration of PET and CT which would allow simultaneous data acquisition is inherently difficult, requiring detector technology capable of the isochronal acquisition of low-energy, high-flux x-rays for CT as well as $511-\mathrm{keV}$ annihilation $\gamma$-rays for PET, investigations into PET/MRI were usually driven by the possibility of truly simultaneous PET and MRI scanning (18).

Acquiring PET and MRI data simultaneously allows essentially perfect temporal correlation of dynamically acquired data sets from both modalities. This could be of special interest for brain imaging as well as in the fields of cardiology and oncology. Basically, MRI already provides a large variety of protocols which selectively enhance contrast and thus visual discrimination among different tissues in vivo, and which can be used for dynamic contrastenhanced imaging, diffusion imaging, functional MRI (fMRI) etc. Thus, PET/MRI could provide MRI-derived information on both anatomy and function for correlation with PETderived pathology-specific, quantitative information on other aspects of tissue function. 
Perhaps most importantly, MR is also capable of spectroscopy for detection of organ-specific abnormalities and pathologies by quantifying ratios of concentrations of molecules such as lactate or N-acetylaspartate (NAA) $(19,20)$. Functional MRI (fMRI) is of special interest in the fields of neurology and psychiatry, since it assesses brain function by detecting a contrast dependent on the blood oxygenation level (BOLD effect) and this on a combination of perfusion as well as oxygenation. To make use of the potential of combined PET and MRI to reveal such multi-functional information as well as anatomy in a single patient examination, any mutual interference between the two imaging modalities needs to be avoided to allow each of the two modalities to perform to their optimum capabilities.

\section{TECHNICAL CHALLENGES OF COMBINED PET AND MRI}

Combining two advanced imaging technologies without degrading the original optimum performance if either is challenging. PET and MRI are two such modalities which route and otherwise process electronic signal pulses which are prone to distortion. Besides avoiding any such signal distortion and associated degradation of performance when combining PET and MRI, the main challenges of merging the hardware into a single device are space constraints and the fact that conventional PET detectors (21) are based on photomultiplier tubes (PMTs), which do not operate properly in presence of a magnetic field (Fig 1).

\section{DIFFERENT APPROACHES FOR COMBINED PET AND MR IMAGING}

There are different conceivable options for combining PET and MR. The easiest and most straight forward approach would be the placement of the two scanners in series in a manner analogous to current PET/CT scanners(Fig. 2a). However, this approach would require significant modifications, especially of the PET detectors to make them insensitive to the magnetic fields and to construct them in such a way as to negligibly affect the performance of the MRI scanner. As with the approach to PET/CT, synchronous temporally correlated data acquisition would not be feasible and thus the potential of such a scanner, especially for novel research applications, would be drastically restricted. In addition, since PET and MRI are relatively slow imaging modalities, the patient examination time would be considerably longer than for simultaneous PET/MRI. Therefore, the preferable approach is clearly full integration of the PET hardware into the MRI gantry (Fig. 2 b, c).

The basic issue is the mutual interference between the two modalities. Such interference would need to be minimized to ensure stable, high-quality PET and MRI operation. The PET subsystem can interfere with the performance of the MRI sub-system by degrading the homogeneity of the MRI's main magnetic field $\left(\mathrm{B}_{0}\right)$ and the radio frequency $(\mathrm{RF})$ field $\left(\mathrm{B}_{1}\right)$ due to the various hardware components of the PET detector. Such interference causes a loss of image quality and is more significant when the fabrication materials have a magnetic susceptibility which differs markedly from that of human tissue. The variable MR gradients may induce eddy currents in conductive materials of the PET detector, which distort the effective applied gradient field. There might also be interactions of the PET electronics with the MR RF field, which could cause artifacts in the MR images. On the other hand, there are also various effects of the MR sub-system on the performance of the PET sub-system. As discussed earlier, the high magnetic field used in MRI excludes the use of PMTs employed in traditional PET scanners. The path of the electrons between the dynodes in the vacuum tube of the PMT is deflected from its normal trajectory by the interaction with the strong external magnetic field due to the Lorentz force. In addition, the RF fields and also the gradient system pulses of the MRI can adversely affect the PET electronics; such effects can range from producing image artifacts due to signal distortion to actual destruction of sensitive PET electronics. 
Space constraints are also a major restriction, since the bore size of MR scanners is limited. The challenge is to design a PET insert that can be placed in or integrated into the MR tunnel with adequate space for a reasonably comfortable patient palette. These space constraints lead also to the fact that a rotating transmission source cannot be used for attenuation correction $(22,23)$. PET attenuation correction therefore has to be done based on the MR image data.

To overcome the foregoing problems, different methods have been proposed and implemented by various groups working. One classification of these different approaches is based on the method employed for solving the problem of the sensitivity of PMTs to magnetic fields, one of the key PET issues, in PET/MRI.

\section{Optical fibers}

The standard approach to solving the PMT-related problem of mutual interference between PET and MRI is to employ light fibers that are coupled to the PET detector's scintillation crystals (Fig. 3a). Therefore, only the $x$ - and $\gamma$-ray detection elements remain in the magnetic field of the MR scanner and the scintillations are directed out of this high magnetic field by the light fibers. Studies have shown that bismuth germanate (BGO) and lutetium orthooxysilicate (LSO) crystals produce only small magnetic distortion, whereas gadolinium orthosilicate (GSO) and lutetium gadolinium orthosilicate (LGSO) have different magnetic susceptibilities compared to human tissue and produce significant distortion and artifacts in MR images (24). Such a system was used in the early PET/MR experiments by Shao et al., who built a single-slice PET scanner consisting of 48 LSO crystals mounted in a 38-mm annular configuration. The scintillations were directed via $4-\mathrm{m}$ long by $2-\mathrm{mm}$ thick optical fibers to three mu-metal-shielded multi-channel PMTs located outside the fringe magnetic field.. Although only a 0.2-T open MR scanner was used, it was still challenging to identify PMTs that could operate reliably in a stray field of less than $10 \mathrm{mT} 3 \mathrm{~m}$ away from the magnet (25). Subsequent studies on a similar system showed on no major PET or MR artifacts and no degradation of PET or MR image quality (26). Despite the apparent lack of mutual interference in a PET/MR design based in optical fibers, such a design has certain shortcomings. Since each scintillation crystal element has to be connected to a light fiber; a huge number of fibers are needed, a number which will increase as the number of detectors increases. This ultimately constrains the axial field of view of light fiber-based PET scanners, since the space inside the MR system is very limited. Moreover, the use of optical fibers results in some loss of light signal compared to the original amount available at the crystal's readout surface. This causes some degradation of timing and energy resolution and of overall PET-system performance (27). Finally, the assembly of a fiber based PET system is not trivial, since optical fibers are usually not easy to bend without introducing an additional light loss. Nevertheless, there are several prototype systems extant based on this approach (28-32). A group at the King's College (London) has proposed building a PET/MR system using concentric detector rings, which provides improved sampling at the center of the field of view. This system will provide depth of interaction (DOI) information and uses optical fibers to direct light from the crystals to multi channel PMTs (32). Raylman et al. have shown in vivo results for a rat from a MRI-compatible small- animal PET system that uses two PET block detectors inside a 3-T clinical MR scanner. The PET detector blocks are connected to 2.5-m long optical fibers to direct the light out of the magnet (30). To achieve state-of-the-art transmission imaging with this system, the two PET detectors are rotated to compensate for the inter-detector gaps.

\section{Semiconductor light detectors}

An alternative approach to PET/MRI is replacing the PMTs with solid-state scintillation detectors such as avalanche photo diodes (APDs) (Fig. 1, Fig. 3) (33). APDs, compared to PMTs, have a lower gain and are more sensitive to temperature variations. However, the major advantage of APDs is that they are insensitive to magnetic fields, so that they can be directly 
connected to the scintillation crystal block within the magnetic field via a short 1- to 2-mm short light guide (34). Therefore, light loss is minimized and the conversion of light to electronic signals occurs inside the MR sub-system, making the use of fragile and bulky optical fibers unnecessary. An additional advantage is that APDs are smaller than PMTs and therefore a more compact design can be achieved, leading to greater flexibility in possibly expanding the axial and transaxial fields of view of the PET sub-system. Accordingly, multiple rows of APD-based detectors can be placed next to each other, making a large axial field of view (particularly important for whole-body imaging) inside the MR sub-system feasible. The low gain of APDs requires charge-sensitive preamplifiers mounted proximal to the APDs to amplify the lowamplitude analog signals. It is therefore critically important to carefully shield the PET subsystem components inside the magnet from electrical interference by the MR scanner. this can be achieved using thin copper shielding, which also helps prevent distortion of MR images by the PET electronic signals. Conductive structures such as those formed by the PET shielding and the circuit boards are prone to the induction of currents caused by the MR gradient system during image acquisition. Eddy currents that would build up in the copper shielding may lead to MR image distortions and also to a reduction of the effective gradient strength and possible heat generation inside the shielding material. Therefore, the design of a fully integrated PET insert using solid-state light detectors remains a challenging task requiring careful consideration of electronic and mechanical components.

Our group has recently shown the feasibility of such a design, successfully fabricating a smallanimal PET/MR system and performing in vivo imaging experiments with this system (Figures 4 and 5) (35). Detailed measurements performed inside and outside the MR scanner have shown PET spatial resolution of less than $2 \mathrm{~mm}$ and system sensitivity of $0.25 \%$; these values not vary whether the PET sub-system, is operated inside or outside the MR sub-system. Moreover, the degradation of homogeneity and signal-to-noise-ratio of a MR phantom scan was on average less than $6 \%$ when the PET insert was in the MR scanner compared to measurements without the PET insert in place. In addition to animal systems, the first human PET/MRI system, for brain imaging, is based on APDs (36) (Fig. 6). In the future, new semiconductor based light detectors, such as Geiger-mode APDs (also known as silicon PMTs) will likely be developed.

\section{Optical fibers and semiconductor light detectors}

Simon Cherry and co-workers at the University of California ( Davis, CA) have developed a PET/MRI system that uses a combination of the two aforementioned techniques (Fig. 3b). A short optical-fiber bundle, $10 \mathrm{~cm}$ in length, directs light from the LSO crystals to position sensitive APDs, which are mounted just outside the field of view of the MR scanner. No major negative interaction between the two sub-systems was observed and simultaneous PET/MR images were successfully acquired (Fig $3 b$ ) (27). This design has the advantages that no metallic components is placed directly in the MR field of view and that the short light guides do not exhibit as much loss of light as extended optical fibers. Thus, the energy resolution of the PET detectors at $511 \mathrm{keV}$ is $25.5 \%$ and the time resolution is $25 \mathrm{~ns}$. However, with such a hybrid approach, the problem of a possible mutual interaction is still present, especially via gradient fringe fields and RF signals, despite the positioning the metallic components outside the MR field of view. Moreover, the axial field of view of such a system is again limited by the number of light fibers that are needed.

\section{Split Magnet}

Another approach to combining PET and MR has been pursued by Lucas et al. (37), who assembled a modified commercial PET system (38) within a gap in a novel magnet design (Fig. $3 \mathrm{c}$ ). The magnet is superconducting and has a field strength of $1 \mathrm{~T}$. In the middle of the magnet is an 80-mm gap, which allows the assembly of a multi-ring PET detector using $120-\mathrm{cm}$ long optical fibers. These light guides connect the LSO crystals of the PET detector ring, residing 
inside the MR subs-system, to position-sensitive PMTs operating within soft iron shielding outside the magnet. In contrast to other fiber-based approaches, the fibers in this design are led outside the MRI subs-system in a radial rather than an axial direction. The magnetic field is reduced at the position of the PMTs to $1 \mathrm{mT}$. However, only $40 \%$ of the original light is transferred through the optical fibers, again resulting in degradation of energy and time resolution. Tests showed, however, that simultaneous PET and MR imaging could be successfully performed with this system (39). In addition to the light loss, the magnetic field strength is limited with a split-magnet design, and higher field-strength systems $(>4.7 \mathrm{~T}$ ) are particularly desirable for small-animal imaging. Furthermore, the homogeneity of the main magnetic field is more difficult to achieve with a split magnet design, which might limit the gradient performance of such a system.

\section{Field Cycling}

MRI using field-cycling schemes differs from conventional MRI. The basic concept is that the static magnetic field is replaced by two dynamically controlled fields. The first of these, the polarizing field, is usually $\sim 1 \mathrm{~T}$ and is used to build up the magnetization inside the scanned object. The polarizing field is switched on only for a short period of time, $\sim 1 \mathrm{~s}$, and then rapidly turned off. Subsequently, a second magnetic field, the "readout" field, is switched on. This second field is much smaller, usually only $\sim 0.1 \mathrm{~T}$. The MR image is acquired when the readout field is on. One of the advantages of field-cycled MR is that the polarizing field need not to fulfill the stringent homogeneity criterion which is typically needed in MRI generally. In general, the technical demands for such a system, in comparison to those for a conventional MR system, are less onerous and the ability to vary the field strength and duration can yield better MR contrast. A small-animal MR scanner based on field cycling has been proposed by Gilbert et al (40). The same group also suggested the combination of PET and field-cycled MR (Fig. 3d). This concept is based on the supposition that a PET sub-system can be installed in the gap of the polarizing magnet. PET and MR data acquisition would be sequential, that is, the PET data are only acquired during when both magnetic fields are off. This has the advantages that no optical fibers are needed and that PMTs can be installed in the vicinity of the scintillation crystals, avoiding excessive light loss (41). Moreover, with such intermittent PET and MR data acquisition, it is difficult to monitor temporal biological processes; as truly simultaneous data acquisition is not possible. Also, because of the relatively high magnetic fields switching at a high frequency, it remains unclear whether the PMT performance would degrade over time. This approach needs further evaluation to identify its full potential as well as all of the associated technical challenges.

\section{APPLICATIONS OF COMBINED PET/MRI}

Considering the fact that the full integration of PET/MRI is technically feasible without compromising the performance of either of the individual modalities, PET/MRI offers considerable potential for novel imaging applications far beyond simply correlation of functional and anatomic images. First, performance evaluation tests have shown that the combination of PET/MRI allows simultaneous acquisition of multi-functional data such as PET tracer uptake, MR spectroscopy. or fMRI along with high-resolution anatomic MR imaging. However, besides the development of novel technology, advanced molecular imaging in pre-clinical research and clinical diagnosis requires appropriate biomarkers and imaging probes. There will be a need to develop multi-modality probes for PET/MRI capable of providing functional information (eg related to cell-cell interactions) well beyond that derivable with the current generation of imaging probes Although the possible combinations of PET/MR imaging protocols is virtually unlimited, and despite the excellent soft-tissue contrast of MRI, there are notable applications of PET/CT (such as lung and bone imaging) which likely could 
not be replaced by PET/MRI. In addition, a major disadvantage of PET/MRI might be its longer acquisition time and higher costs compared to those for current PET/CT technology.

\section{PET/MRI IN PRECLINICAL RESEARCH}

High-resolution small-animal CT exposes the rodents to a significantly high radiation doses than those associated with clinical CT and, for vascular contrast-based imaging, requires relatively large amounts of dedicated iodine-based media to improve visualization and discrimination of soft tissues. Thus, other than for bone and lung, CT is generally not the modality of choice for anatomic imaging of small laboratory animals. Combining PET/MRI, thereby providing marked soft-tissue contrast without a potentially excessive radiation exposure, is therefore logical for pre-clinical studies

Encouraging results in the field of oncology have already been achieved using our 7-T PET/ MRI animal scanner (35). Here, the MRI sub-system incorporates a MR-compatible PET insert (Fig 4). This set-up was successfully used for imaging a mouse bearing a CT26 colon tumor. Prior to the scan, the mouse was injected with 13.9 MBq of the proliferation radiotracer 3'$\left[{ }^{18} \mathrm{~F}\right]$ fluoro-3'-deoxy-thymidine $\left(\left[{ }^{18} \mathrm{~F}\right] \mathrm{FLT}\right)$, and a tracer uptake period of 120 minutes, during which the mouse was awake, was used. A catheter was placed in the lateral tail vein of the mouse, thereby allowing administration of various probes and contrast media while the animal was positioned in the combined PET/MRI scanner under isoflurane anesthesia. The PET images were acquired at the same time as the pre- and then the post-contrast-media MR images were obtained. The total MR imaging time was 34 minutes; the PET data were acquired for 45 minutes. No attenuation correction was applied. Regions of interest (ROIs) were drawn in the MR images, which showed increased contrast enhancement and a rapid rise in those areas of the tumor have high FLT uptake and a slow rise in those tumor areas having low FLT uptake, the latter presumably identifying necrotic areas in the core of the tumor. These finding were confirmed histologically. Tumor necrotic could thus be clearly identified by interpreting the MR contrast time-signal curves; the PET images alone would not have disclosed such information.

\section{CLINICAL PET/MRI}

Although all initial PET/MRI work was based on small-animal imagers, many clinicians see a promising future for PET/MRI. At least one manufacturer of medical imaging equipment is focusing on combining PET and MRI in a single device capable of simultaneous data acquisition. As a first step, a PET scanner based on LSO scintillation crystals and APDs was developed to be inserted into the imaging tunnel of a 3-T clinical MRI system (Fig. 5). Initial performance testing on phantoms as well as the first PET/MRI studies in man confirmed an excellent, unaltered performance of both the PET and the MRI - even when operated simultaneously $(36,42)$. Results of a first FDG PET and MRI study of a rat, performed at the site of the first installation site of the human brain PET insert in Tübingen, Germany, are shown in Figure 6. These simultaneously acquired PET/MRI data show good overall system performance. Of course, further detailed evaluation tests will be performed before this scanner is used to examine patients.

The fact that today all PET scanners are sold as part of a combined PET/CT device demonstrates clearly the value of comprehensive functional and morphological information, especially in the field of oncology as well as in neurology or cardiology. PET/MRI will allow elimination of the CT x-ray dose, which generally accounts for most of the dose associated with a PET/ CT scan (15). With PET/MRI, therefore, clinicians might be more amenable to follow-up studies and screening. Thus, PET/MRI might emerge as the modality of choice in clinical oncology. One recent study demonstrated in some cases the diagnostic advantages of fused PET and MR images over PET/CT (43). With the ability of PET/MRI to obtain multi- 
parameters functional data, kinetic studies in neurology and psychiatry can be advanced by correlating the dynamic PET tracer or drug distribution in various brain structures with flowdependent kinetics measured by MRI. Also, effects of transmitter or receptor activation on perfusion might be investigated by a single PET/MRI examination. We anticipate exciting studies in the field of neurology and psychiatry focusing on simultaneous activation studies by PET and fMRI.

\section{PET ATTENUATION CORRECTION BASED ON MRI}

To achieve an accurate activity quantitation in PET, attenuation correction must be applied to the emission data to compensate for the absorption of $x$ - and $\gamma$-rays (44). In PET/CT, this is usually corrected by using a CT-derived attenuation-factor map (45). This is feasible since CT is based on a measure of $\mathrm{x}$-ray attenuation, which is related to electron density of the stopping medium. MR, on the other hand, provides semi-quantitative information on proton density. Thus, PET attenuation correction based only on MRI data is challenging and several groups are focusing on this topic. Acquiring transmission data as was done in PET-only scanners, by using a rotating $\gamma$-ray - or positron source, would be problematic in a PET/MR scanner because of space constraints in the MRI tunnel and prolongation of the overall acquisition time.

The main problem in MR-based PET attenuation correction, however, is that attenuation is not directly correlated to the signal measured by MR. The most obvious examples are bone compacta and air: in MRI both show the same (essentially zero) MRI signal with standard sequences while $511 \mathrm{keV} \gamma$-ray attenuation values of bone and air, respectively, are widely divergent.. Currently, there are two major approaches to solving this problem. The first approach is based on a two- or three-component segmentation of the MR images, using atlas information to correlate known attenuation values to anatomical structures thus segmented. The disadvantage of this procedure is that atlases do not reflect inter-subject anatomic variability, which might make correlation between anatomic structures and the MRI segments problematic. Distortion artifacts or truncation of organs outside the MR field of view, like arms, cannot be accounted for by this method. Our laboratory is currently pursuing another approach for MR imaging-based attenuation correction, a combination of atlas registration and local pattern recognition to capture global variations and predict attenuation maps purely based on MR information. This technique is implemented using a machine-learning approach $(46,47)$. We applied this correction method to PET data for human heads and for which we also had MR images. Errors in lesion activity quantitation, compared to values based on CT attenuation correction, were generally less than $10 \%$. Initial results showed that this approach may also be applicable to whole-body images.

\section{CONCLUSION}

The initial results achieved with various prototype animal PET/MRI systems as well as the first clinical PET/MRI brain-imaging devices demonstrate the feasibility of simultaneous data acquisition with negligible mutual interference between the two sub-systems. The potential of fully integrated PET/MRI goes far beyond simple registration of morphological and functional images and holds the promise of temporal as well as spatial correlation of multi-parameter functional data derived by MR spectroscopy and functional imaging with functional PET data. The installation of the first human PET/MRI scanner is a clear harbinger that this new multimodality technology will soon impact clinical practice, perhaps even more dramatically than $\mathrm{PET} / \mathrm{CT}$.

\section{REFERENCES}

1. Beyer T, Townsend DW, Brun T, et al. A combined PET/CT scanner for clinical oncology. J Nucl Med 2000;41:1369-1379. [PubMed: 10945530] 
2. Townsend DW. A combined PET/CT scanner: the choices. J Nucl Med 2001;42:533-534. [PubMed: 11337536]

3. Antoch G, Saoudi N, Kuehl H, et al. Accuracy of whole-body dual-modality fluorine-18-2-fluoro-2deoxy-D-glucose positron emission tomography and computed tomography (FDG-PET/CT) for tumor staging in solid tumors: comparison with CT and PET. J Clin Oncol 2004;22:4357-4368. [PubMed: 15514377]

4. Bar-Shalom R, Yefremov N, Guralnik L, et al. Clinical performance of PET/CT in evaluation of cancer: additional value for diagnostic imaging and patient management. J Nucl Med 2003;44:1200-1209. [PubMed: 12902408]

5. Scarfone C, Lavely WC, Cmelak AJ, et al. Prospective feasibility trial of radiotherapy target definition for head and neck cancer using 3-dimensional PET and CT imaging. J Nucl Med 2004;45:543-552. [PubMed: 15073248]

6. Herrmann T. Radiation oncology and functional imaging. Nuklearmedizin 2005;44(Suppl 1):S38-40. [PubMed: 16395977]

7. Pfannenberg AC, Aschoff P, Brechtel K, et al. Value of contrast-enhanced multiphase CT in combined PET/CT protocols for oncological imaging. Br J Radiol. 2007

8. Beyer T, Antoch G, Blodgett T, Freudenberg LF, Akhurst T, Mueller S. Dual-modality PET/CT imaging: the effect of respiratory motion on combined image quality in clinical oncology. Eur J Nucl Med Mol Imaging 2003;30:588-596. [PubMed: 12582813]

9. Brechtel K, Klein M, Vogel M, et al. Optimized contrast-enhanced CT protocols for diagnostic wholebody 18F-FDG PET/CT: technical aspects of single-phase versus multiphase CT imaging. J Nucl Med 2006;47:470-476. [PubMed: 16513616]

10. Goerres GW, Burger C, Schwitter MR, Heidelberg TN, Seifert B, von Schulthess GK. PET/CT of the abdomen: optimizing the patient breathing pattern. Eur Radiol 2003;13:734-739. [PubMed: 12664111]

11. Goerres GW, Kamel E, Heidelberg TN, Schwitter MR, Burger C, von Schulthess GK. PET-CT image co-registration in the thorax: influence of respiration. Eur J Nucl Med Mol Imaging 2002;29:351360. [PubMed: 12002710]

12. Barentsz JO, Jager GJ, Witjes JA, Ruijs JH. Primary staging of urinary bladder carcinoma: the role of MRI and a comparison with CT. Eur Radiol 1996;6:129-133. [PubMed: 8797968]

13. Kent DL, Haynor DR, Longstreth WT Jr. Larson EB. The clinical efficacy of magnetic resonance imaging in neuroimaging. Ann Intern Med 1994;120:856-871. [PubMed: 7818632]

14. Prabhakar R, Haresh KP, Ganesh T, Joshi RC, Julka PK, Rath GK. Comparison of computed tomography and magnetic resonance based target volume in brain tumors. J Cancer Res Ther 2007;3:121-123. [PubMed: 17998738]

15. Brix G, Lechel U, Glatting G, et al. Radiation exposure of patients undergoing whole-body dualmodality 18F-FDG PET/CT examinations. J Nucl Med 2005;46:608-613. [PubMed: 15809483]

16. Cherry SR, Shao Y, Siegel S, et al. Optical fiber readout of scintillator arrays using a multi-channel PMT: a high resolution PET detector for animal imaging. Nuclear Science, IEEE Transactions on 1996;43:1932-1937.

17. Pichler BJ, Judenhofer MS, Catana C, et al. Performance test of an LSO-APD detector in a 7-T MRI scanner for simultaneous PET/MRI. J Nucl Med 2006;47:639-647. [PubMed: 16595498]

18. Nassalski, A.; Nassalski, A.; Moszynski, M.; Szczesniak, T.; Wolski, DAWD.; Batsch, TABT. The Road to the Common PET/CT Detector. In: Moszynski, M., editor. Nuclear Science Symposium Conference Record, 2006. IEEE; 2006. p. 1904The Road to the Common PET/CT Detector

19. Pfeuffer J, Juchem C, Merkle H, Nauerth A, Logothetis NK. High-field localized 1H NMR spectroscopy in the anesthetized and in the awake monkey. Magn Reson Imaging 2004;22:13611372. [PubMed: 15707786]

20. Tkac I, Henry PG, Andersen P, Keene CD, Low WC, Gruetter R. Highly resolved in vivo 1H NMR spectroscopy of the mouse brain at 9.4 T. Magn Reson Med 2004;52:478-484. [PubMed: 15334565]

21. Casey ME, Nutt R. A multicrystal two dimensional BGO detector system for positron emission tomography. IEEE Trans Nucl Sci 1986;33:460-463.

22. Ostertag H, Kubler WK, Doll J, Lorenz WJ. Measured attenuation correction methods. Eur J Nucl Med 1989;15:722-726. [PubMed: 2583200] 
23. deKemp RA, Nahmias C. Attenuation correction in PET using single photon transmission measurement. Med Phys 1994;21:771-778. [PubMed: 7935213]

24. Yamamoto S, Kuroda K, Senda M. Scintillator selection for MR-compatible gamma detectors. Nuclear Science, IEEE Transactions on 2003;50:1683-1685.

25. Shao Y, Cherry SR, Farahani K, et al. Simultaneous PET and MR imaging. Phys Med Biol 1997;42:1965-1970. [PubMed: 9364592]

26. Slates RB, Farahani K, Shao Y, et al. A study of artefacts in simultaneous PET and MR imaging using a prototype MR compatible pet scanner. Phys Med Biol 1999;44:2015-2027. [PubMed: 10473211]

27. Catana C, Wu Y, Judenhofer MS, Qi J, Pichler BJ, Cherry SR. Simultaneous Acquisition of Multislice PET and MR Images: Initial Results with a MR-Compatible PET Scanner. J Nucl Med 2006;47:19681976. [PubMed: 17138739]

28. Yamamoto S, Mazumoto K, Senda M. Development of a multi-slice dual layer MR-compatible animal PET system using DOI detectors. Supplement to the Journal of Nuclear Medicine 2007;48:89P.

29. Shao Y, Cherry SR, Farahani K, et al. Development of a PET detector system compatible with MRI/ NMR systems. Nuclear Science, IEEE Transactions on 1997;44:1167-1171.

30. Raylman RR, Majewski S, Lemieux SK, et al. Simultaneous MRI and PET imaging of a rat brain. Phys Med Biol 2006;51:6371-6379. [PubMed: 17148823]

31. Marsden PK, Strul D, Keevil SF, Williams SC, Cash D. Simultaneous PET and NMR. Br J Radiol 2002;75 Spec No:S53-59. [PubMed: 12519736]

32. Mackewn JE, Strul D, Hallett WA, et al. Design and development of an MR-compatible PET scanner for imaging small animals. Nuclear Science, IEEE Transactions on 2005;52:1376.

33. Pichler BJ, Lorenz E, Mirzoyan R, et al. Performance test of a LSO-APD PET module in a 9.4 Tesla magnet. IEEE Nuclear Science Symposium 1997;2:1237-1239.

34. Pichler BJ, Swann BK, Rochelle J, Nutt RE, Cherry SR, Siegel SB. Lutetium oxyorthosilicate block detector readout by avalanche photodiode arrays for high resolution animal PET. Phys Med Biol 2004;49:4305-4319. [PubMed: 15509067]

35. Judenhofer MS, Wehrl HF, Newport DF, et al. Simultaneous PET/MRI: A new Approach for Functional and Morphological Imaging. Nat Med. 2008in press

36. Schmand M, Burbar Z, Corbeil JL, et al. Brain PET: First human tomograph for simultaneous (functional) PET and MR imaging. Supplement to the Journal of Nuclear Medicine 2007;48:45P.

37. Lucas AJ, Hawkes RC, Ansorge RE, et al. Development of a combined microPET-MR system. Technol Cancer Res Treat 2006;5:337-341. [PubMed: 16866564]

38. Tai YC, Ruangma A, Rowland D, et al. Performance evaluation of the microPET focus: a thirdgeneration microPET scanner dedicated to animal imaging. J Nucl Med 2005;46:455-463. [PubMed: 15750159]

39. Lucas A, Hawkes RC, Guerra P, et al. Development of a combined micro-PET-MR system. IEEE Nuclear Science Symposium Conference Record 2006:2345-2348.

40. Gilbert KM, Handler WB, Scholl TJ, Odegaard JW, Chronik BA. Design of field-cycled magnetic resonance systems for small animal imaging. Phys Med Biol 2006;51:2825-2841. [PubMed: 16723769]

41. Handler WB, Gilbert KM, Peng H, Chronik BA. Simulation of scattering and attenuation of $511 \mathrm{keV}$ photons in a combined PET/field-cycled MRI system. Phys Med Biol 2006;51:2479-2491. [PubMed: 16675864]

42. Schlemmer H-P, Pichler BJ, Wienhard K, et al. Simultaneous MR/PET for Brain Imaging: First Patient Scans. Supplement to the Journal of Nuclear Medicine 2007;48:45P.

43. Muller-Horvat C, Radny P, Eigentler TK, et al. Prospective comparison of the impact on treatment decisions of whole-body magnetic resonance imaging and computed tomography in patients with metastatic malignant melanoma. Eur J Cancer 2006;42:342-350. [PubMed: 16364631]

44. Huang SC, Hoffman EJ, Phelps ME, Kuhl DE. Quantitation in positron emission computed tomography: 2. Effects of inaccurate attenuation correction. J Comput Assist Tomogr 1979;3:804814. [PubMed: 315970]

45. Kinahan PE, Townsend DW, Beyer T, Sashin D. Attenuation correction for a combined 3D PET/CT scanner. Med Phys 1998;25:2046-2053. [PubMed: 9800714] 
46. Hofmann, M.; Steinke, F.; Scheel, V., et al. MR-Based PET Attenuation Correction - Method and Validation. IEEE Nuclear Science Symposium and Medical Imaging Conference Issue; 2007.

47. Zien A, Ratsch G, Mika S, Scholkopf B, Lengauer T, Muller KR. Engineering support vector machine kernels that recognize translation initiation sites. Bioinformatics 2000;16:799-807. [PubMed: 11108702] 


\section{Conventional PET detectors}
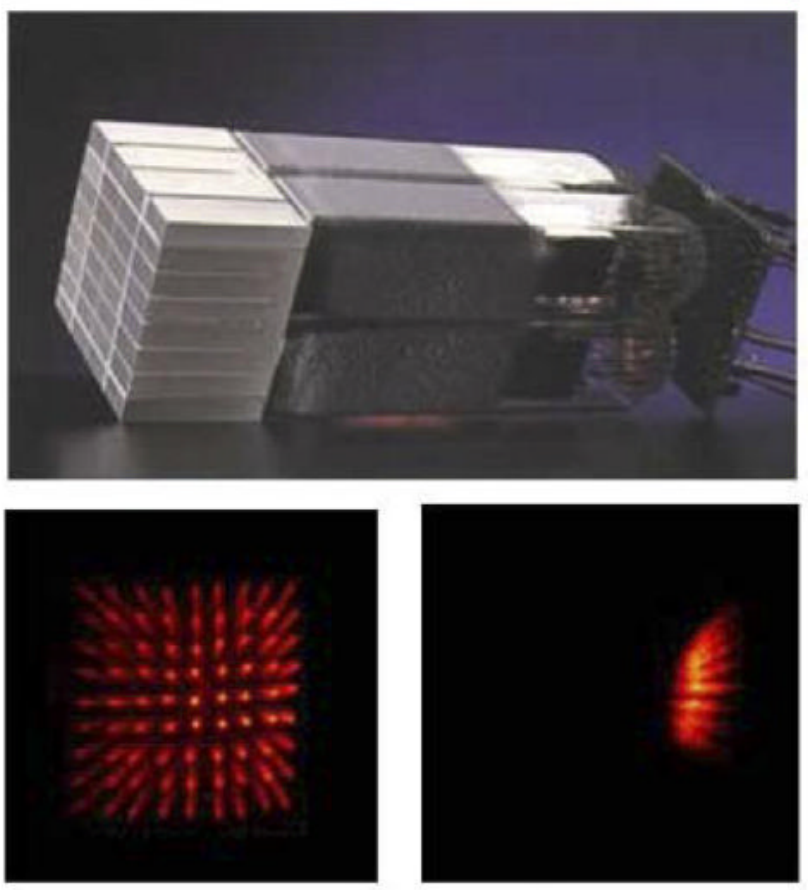

$B=0$

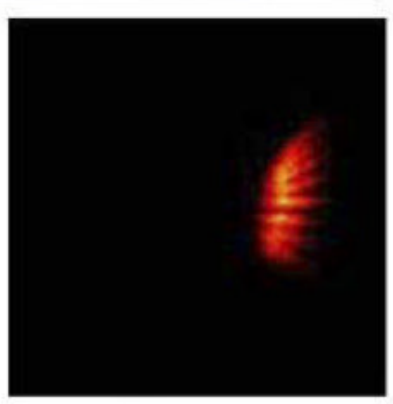

$B \neq 0$

\section{APD-based PET detectors}
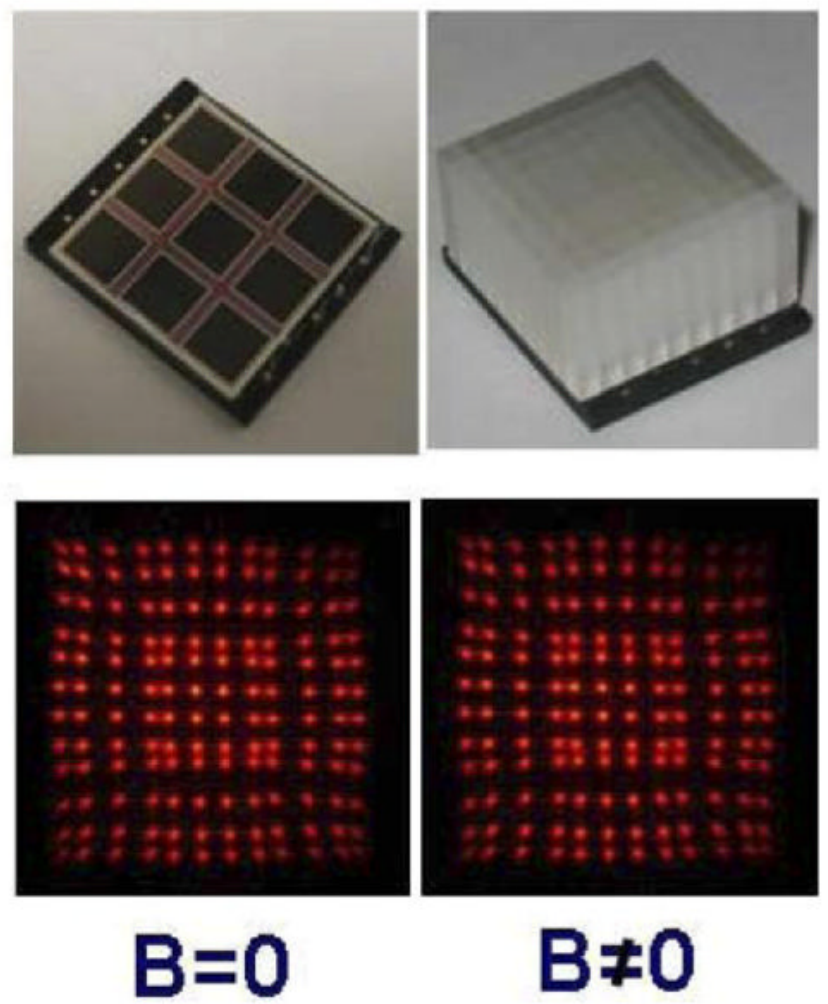

Figure 1.

Conventional PET detectors are based on scintillation crystal blocks read out by photomultiplier tubes (PMTs)which are highly sensitive to even small magnetic fields )B). These block detectors are standard in current clinical PET scanners. The detector-element map of a position-sensitive PMT-based block detector is completely distorted when a small horseshoe magnet is placed near the PMT (left). The detector-element map of APD-based PET detectors remain undistorted even in the presence of a 7-T magnetic field (right). 

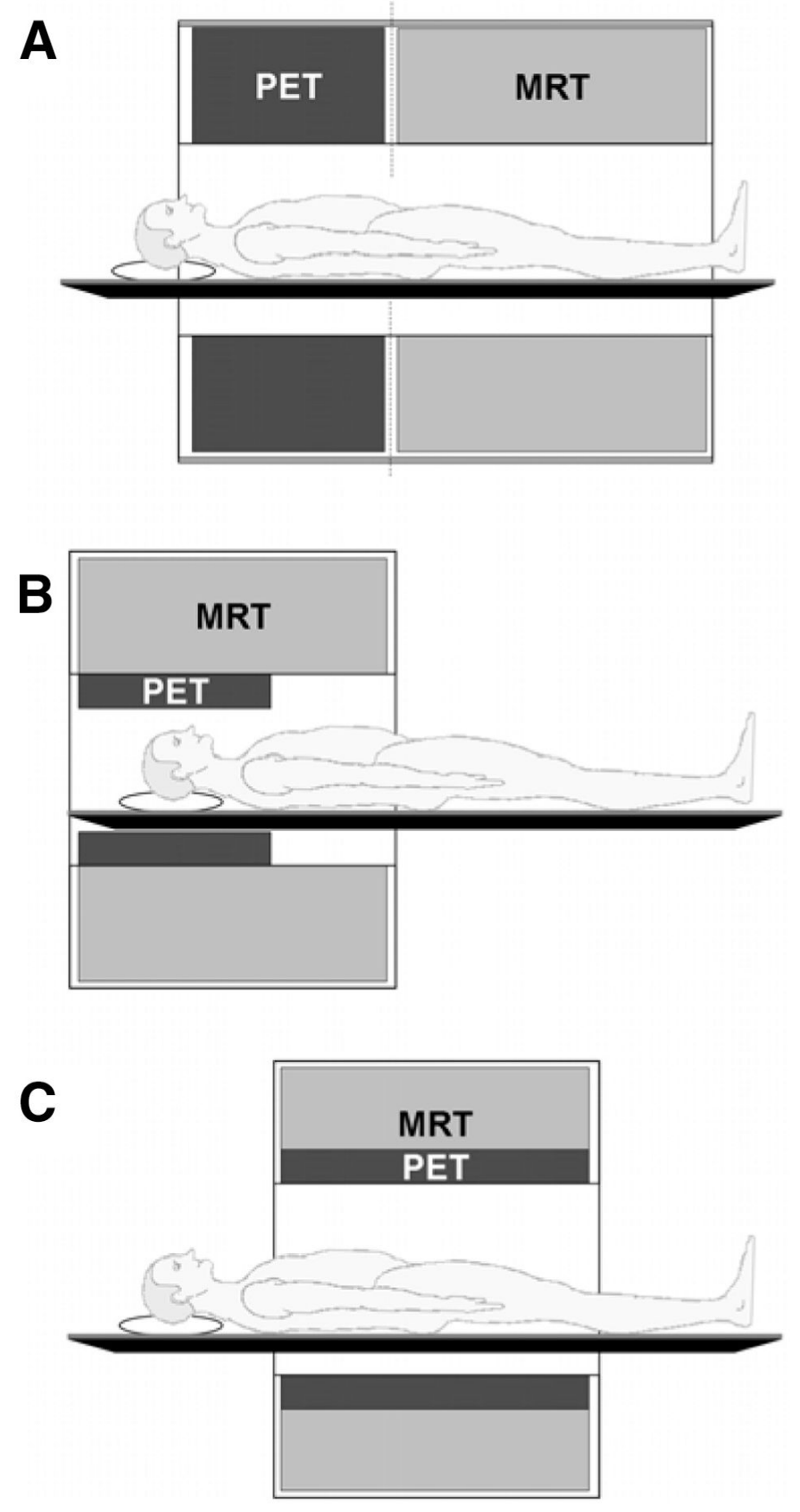

Figure 2.

Potential approaches for combined PET/MRI in a clinical setting. (a) An in-line solution similar to current PET/CT scanners which mechanically combines a standard MRI with a slightly modified PET scanner would be the easiest approach but also offers a limited variety of imaging protocols and would not allow simultaneous PET/MR imaging. In addition, the total imaging time for the sequential PET and MRI scans is prolonged. (b) Schematic diagram of the first commercial clinical PET/MRI scanner. The removable PET detector slip-fits into a standard clinical MRI system. This approach allows simultaneous data acquisition and flexibility in using the MRI scanner as stand-alone device or as a PET/MRI device, but at the cost of a reduced PET/MRI field of view suitable only for brain imaging. (c) The most advanced but 
also the most technically challenging approach, providing the highest degree of flexibility in selecting imaging protocols, would be full integration of the PET detectors into the MRI gantry. 

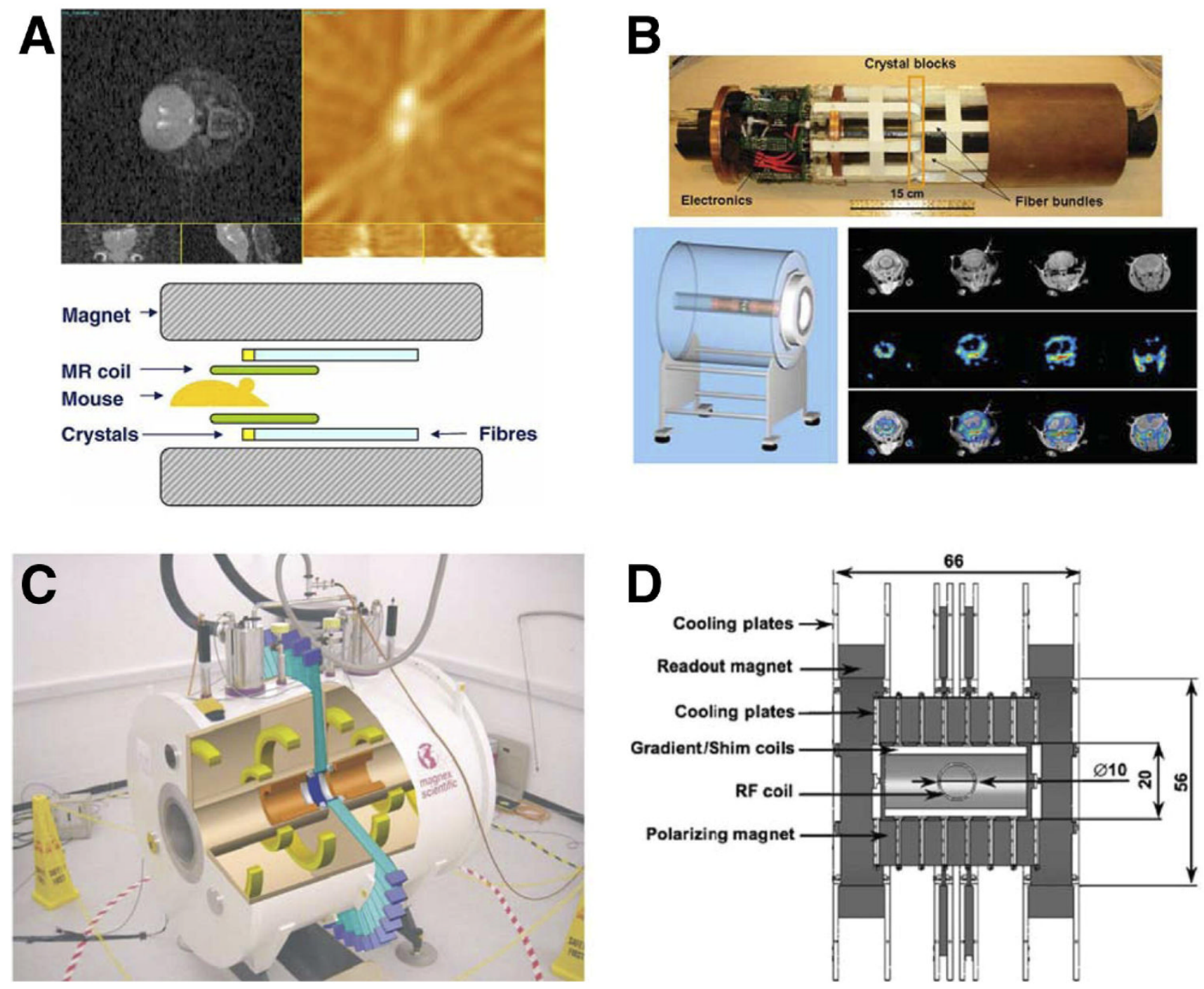

Figure 3.

Different approaches to combined PET/MRI systems which are currently under investigation. (a) One of the first PET/MRI approaches, pursued by Marsden and by Cherry et al. Long light fibers are used to direct the light signal outside the magnetic fringe field. Initial single-slice PET and MRI images of a [ ${ }^{18}$ F]FDG-injected rat acquired with this system (31). (b) The hybrid PET/MRI approach of Catana et al uses shorter light fibers in combination with APDs. Initial images of a $\left[{ }^{18} \mathrm{~F}\right]$ fluoride-injected mouse successfully acquired with this system (27). (c) Splitmagnet PET/MRI. A 1-T magnet is split in two parts, which providing space for the LSO blocks in the gap between the two magnet halves. The light is read-out by radially arranged optical fibers and position-sensitive PMTs (37). (d) Schematic diagram of a field-cycled MR system. The MR sub-system can be shut off during acquisition of PET data (41). 


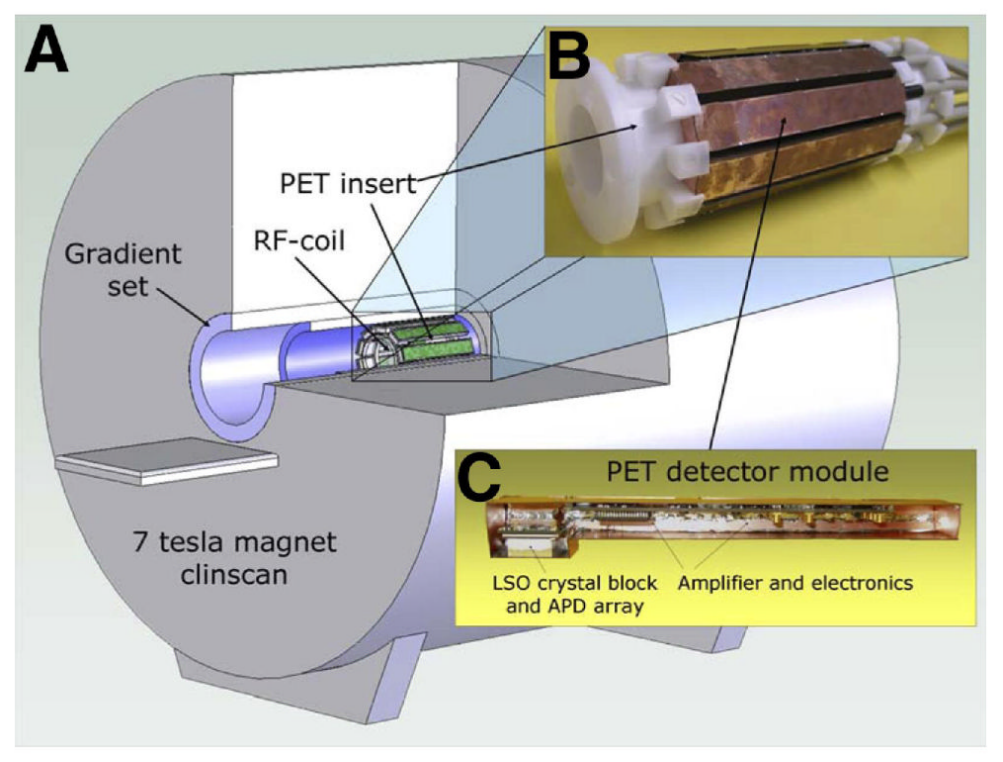

Figure 4.

A combined PET/MRI scanner for simultaneous PET and MR data acquisition. (a) The PET ring is comprised of ten $12 \times 12$ LSO detectors directly coupled to a $3 \times 3$ APD array. (b) All parts of the PET module are non-magnetic. c) The dedicated mouse RF coil fits into the PET detector ring which is then placed inside the gradient set of a 7-T small-animal MRI system (ClinScan, Bruker BioSpin MRI, Germany). The PET insert has an axial FOV of $19 \mathrm{~mm}$ and a transaxial FOV of $35 \mathrm{~mm}$, which is limited by the mouse RF coil. The spatial resolution of the PET insert is between 1.5 and $2 \mathrm{~mm}(35)$. 

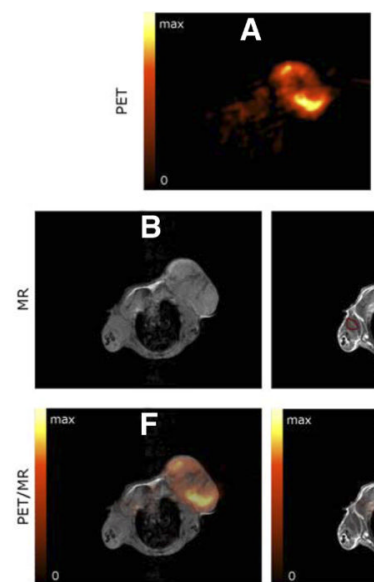
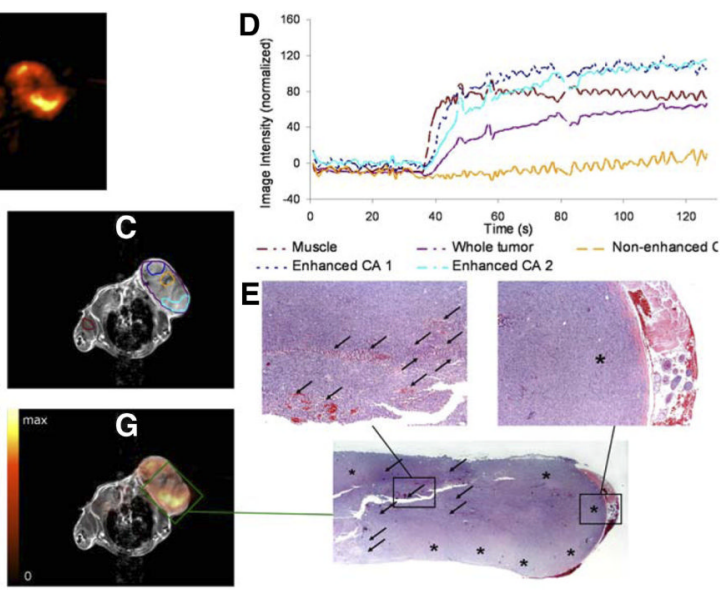

$\begin{array}{ll}-\cdots \text { Muscle } & \text { - W. Whole tumor } \\ \text {-... Enhanced CA } 1 & \text { - . Enhanced CA } 2\end{array}$

E

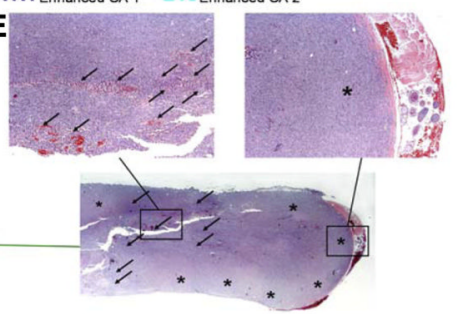

Figure 5.

Results of a PET/MRI animal study using our prototype MR-compatible axial PET scanner built into a 7-T MRI. The functional and morphological data were simultaneously obtained by acquiring PET (a) and pre (b) as well as post (c)-contrast MRI data. The dynamic MR protocols reveal the kinetic of the MR contrast agent (c, d) in tumor areas defined by user-defined ROIs. Tumor areas showing a low uptake of the proliferation marker $\left[{ }^{18} \mathrm{~F}\right] \mathrm{FLT}$ in the PET images $(\mathrm{a}, \mathrm{f}, \mathrm{g})$ correspond to slow, shallow uptake of MR contrast media uptake in the same regions of the tumor, indicative of necrosis. Histology confirms the in vivo findings (e). The hardwarebased fusion accuracy of PET/MR images is better than $0.5 \mathrm{~mm}$ (f, g) (35). 


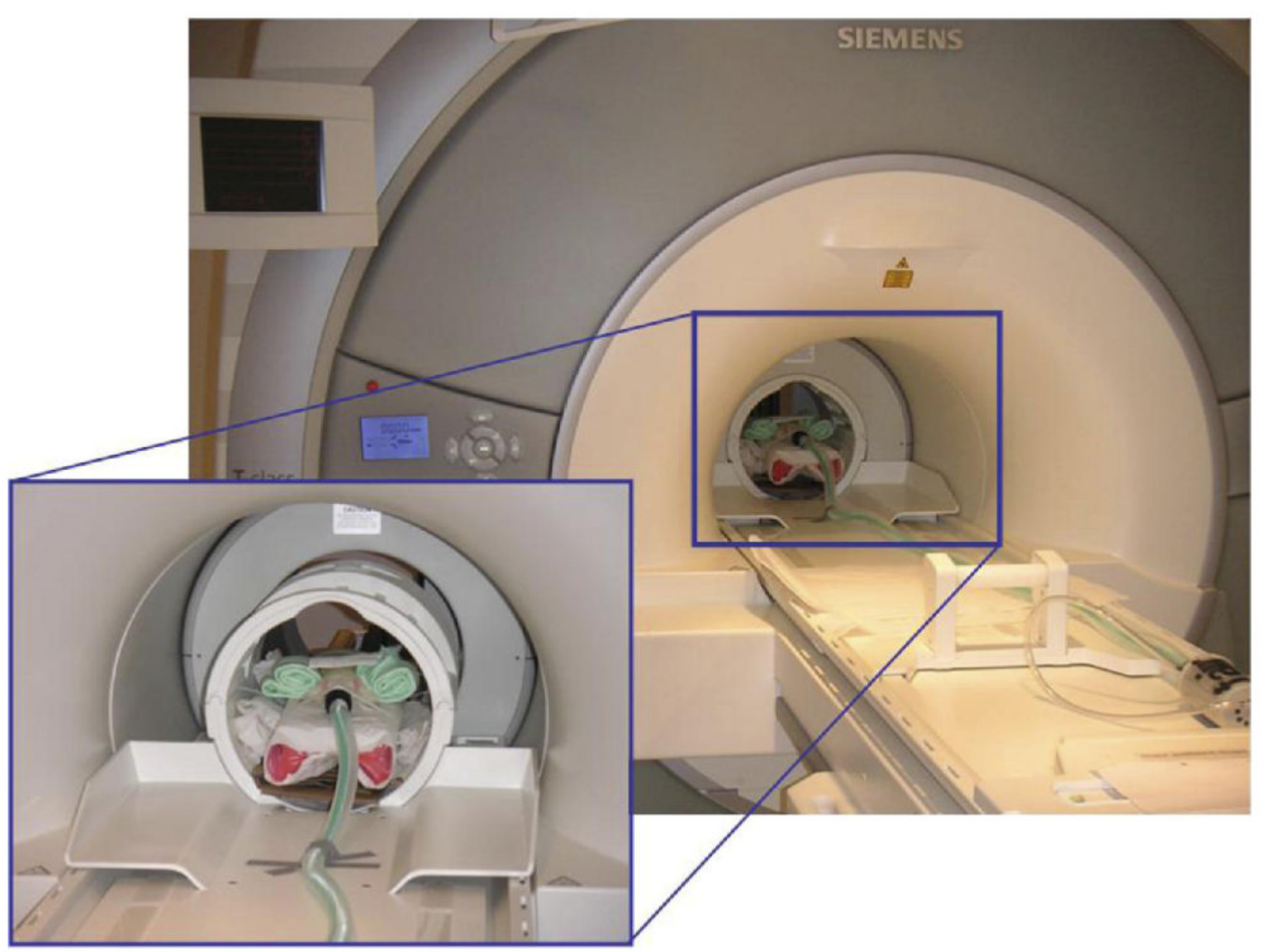

Figure 6.

Installation of the first clinical PET/MRI scanner at the University of Tübingen, (Tubinger, Germany). The removable PET scanner is based on LSO-APD technology and slip-fits into a standard 3-T clinical Magnetom Trio MRI system (Siemens Medical Solutions). The PET insert can be positioned to be operated simultaneously with the MRI or removed so that the MRI system can be used as a stand-alone scanner. This provides maximum flexibility at reduced costs. However, since the PET detectors are physically integrated into the MRI gantry, space constraints allow only imaging of the human head using a dedicated MR head-coil. Currently, this first commercial combined human PET/MRI prototype system is being evaluated using phantoms and animals; plans are to perform the initial patient scans within the several few months. 


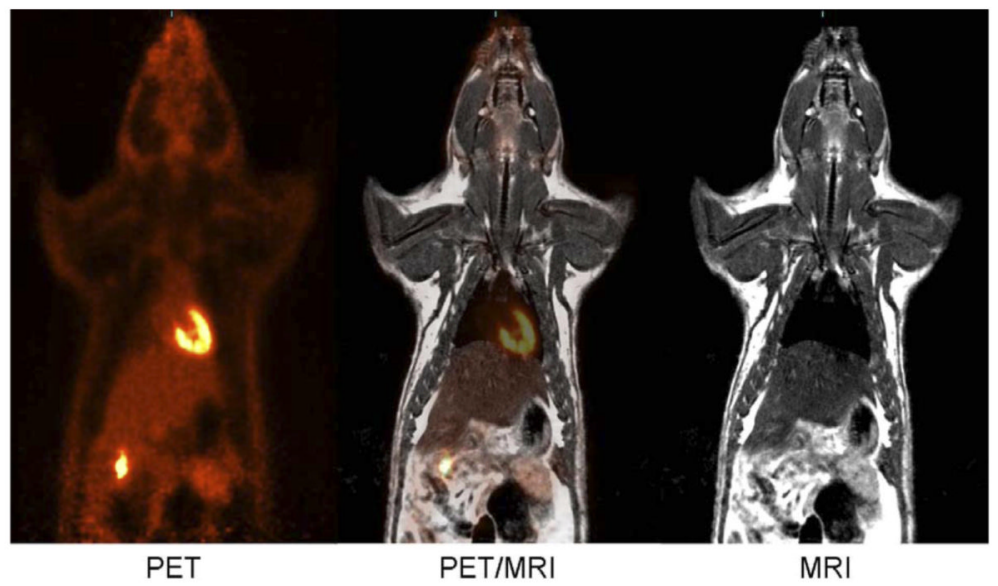

Figure 7.

First PET/MR image of a rat acquired on the human PET/MRI scanner (Siemens Medical Solutions) shown in Figure 6 using a head RF coil. Both the PET and the MRI data were acquired simultaneously. The 400-gram rat was intra-venously injected with $30 \mathrm{MBq}$ of $\left[{ }^{18} \mathrm{~F}\right]$ FDG 30 minutes prior to PET imaging. While PET data acquisition was performed, various MRI sequences were applied to obtain anatomical images. Despite the use of the human head coil, the anatomy of the rat is clearly visualized on the MR images. 